Draft Version April 23, 2022

Preprint typeset using $\mathrm{L}^{A} \mathrm{~T}_{\mathrm{E}} \mathrm{X}$ style emulateapj v. 11/10/09

\title{
MEASURING GAS ACCRETION AND ANGULAR MOMENTUM NEAR SIMULATED SUPERMASSIVE BLACK HOLES
}

\author{
Robyn Levine $^{1}$, Nickolay Y. Gnedin ${ }^{2,3,4}$, And Andrew J. S. Hamilton ${ }^{5,6}$ \\ Draft version April 23, 2022
}

\begin{abstract}
Using cosmological simulations with a dynamic range in excess of $10^{7}$, we study the transport of gas mass and angular momentum through the circumnuclear region of a disk galaxy containing a supermassive black hole (SMBH). The simulations follow fueling over relatively quiescent phases of the galaxy's evolution (no mergers) and without feedback from active galactic nuclei (AGNs), as part of the first stage of using state-of-the-art, high-resolution cosmological simulations to model galaxy and black hole co-evolution. We present results from simulations at different redshifts $(z=6,4$, and 3 ) and three different black hole masses $\left(3 \times 10^{7}, 9 \times 10^{7}\right.$, and $3 \times 10^{8} \mathrm{M}_{\odot}$; at $\left.z=4\right)$, as well as a simulation including a prescription that approximates optically thick cooling in the densest regions. The interior gas mass throughout the circumnuclear disk shows transient and chaotic behavior as a function of time. The Fourier transform of the interior gas mass follows a power law with slope -1 throughout the region, indicating that, in the absence of the effects of galaxy mergers and AGN feedback, mass fluctuations are stochastic with no preferred timescale for accretion over the duration of each simulation $(\sim 1-2 \mathrm{Myr})$. The angular momentum of the gas disk changes direction relative to the disk on kiloparsec scales over timescales less than $1 \mathrm{Myr}$, reflecting the chaotic and transient gas dynamics of the circumnuclear region. Infalling clumps of gas, which are driven inward as a result of the dynamical state of the circumnuclear disk, may play an important role in determining the spin evolution of an SMBH, as has been suggested in stochastic accretion scenarios.

Subject headings: galaxies: evolution—galaxies: high-redshift—galaxies: nuclei-galaxies: structure
\end{abstract}

\section{INTRODUCTION}

Supermassive black holes (SMBHs) with masses from $\sim 10^{6}$ to more than $\sim 10^{9} \mathrm{M}_{\odot}$ are found in the centers of most galaxies (e.g., Kormendy \& Richstone 1995; Magorrian et al. 1998). Observations of correlations between the masses of SMBHs and several properties of their host galaxies indicate that the growth of SMBHs is closely tied to the evolution of their hosts (e.g., Magorrian et al. 1998; Ferrarese \& Merritt 2000; Gebhardt et al. 2000; Tremaine et al. 2002). Scenarios including feedback from active galactic nuclei (AGNs) have been explored in order to understand both the growth of SMBHs and the nature of their relationship with their host galaxies (e.g., Silk \& Rees 1998; Kauffmann \& Haehnelt 2000; Wvithe \& Loeb 2003; Di Matteo et al. 2005; Springel et al. 2005a.b; Croton et al.|2006; Di Matteo et al. 2008; Hopkins et al. 2007; Johansson et al. 2009b). AGNs are thought to be powered by accreting SMBHs (e.g., Lynden-Bell 1969), so that observations of the various phases of AGN activity may provide clues about the connection between SMBH growth and galaxy evolution, as models explored by, e.g., Hopkins et al. (2005) and Sijacki et al. (2007)

\footnotetext{
${ }^{1}$ CITA, 60 St. George St., Toronto, ON, M5S 3H8,Canada; levine@cita.utoronto.ca

2 Particle Astrophysics Center, Fermi National Accelerator Laboratory, Batavia, IL 60510, USA

${ }^{3}$ Kavli Institute for Cosmological Physics, The University of Chicago, Chicago, IL 60637, USA

${ }^{4}$ Department of Astronomy \& Astrophysics, The University of Chicago, Chicago, IL 60637, USA

5 JILA, University of Colorado, Boulder, CO 80309, USA

${ }^{6}$ Department of Astrophysical \& Planetary Sciences, University of Colorado, Boulder, CO 80309, USA
}

have shown. It is therefore essential to characterize accretion onto SMBHs in order to understand AGNs and their relevance for galaxy evolution.

Substantial SMBH growth requires a mechanism for replenishing fuel on the subparsec scales feeding the SMBH accretion disk. Large scale tidal torques caused by galaxy mergers are an effective mechanism for transporting gas from super-galactic and galactic scales down to scales of several parsecs, where other mechanisms may become more viable for funneling material the rest of the distance toward the SMBH (e.g., Hernquist 1989; Barnes \& Hernquist 1992). Secular evolution may also play a role as disk instabilities lead to the formation of bars and spiral waves which can transport material (Roberts et al. 1979; Shlosman et al. 1989; Regan \& Teuben 2004). There are several observations of inflowing gas in the circumnuclear regions of low-luminosity AGNs, apparently driven by global instabilities arising from secular evolution (see, e.g., García-Burillo et al. 2005; Boone et al. 2007; Storchi-Bergmann et al. 2007; Lindt-Krieg et al. 2008; Riffel et al. 2008). Mergers are thought to be effective for building SMBHs in more massive earlytype galaxies, which appear to form earlier (see, e.g., Hopkins et al. 2008a b, and references therein), ultimately building the SMBH-bulge relationships, whereas secular evolution may play a larger role in the growth of late-type galaxies, particularly at lower redshift (see, e.g., Kormendy \& Kennicutt 2004, and references therein).

Whichever is the dominant mechanism for fueling SMBHs, the specific details of how fueling occurs in the circumnuclear regions of galaxies are still not understood. Fueling may occur continuously, over the course 
of large-scale dynamical instabilities in the galaxy, or it may be an intermittent process, dependent entirely on the dynamics on small scales. Cold gas, stochastically accreted from the circumnuclear regions of galaxies, may be able to sustain the fueling of low-luminosity AGNs (Hopkins \& Hernquist 2006). Intermittent accretion episodes, consisting of in-falling clouds of gas with randomly oriented angular momentum vectors, may contribute to the spin-down of SMBHs, thus lowering their radiative efficiency and allowing them to grow faster (see King \& Pringle 2006, 2007; King et al. 2008). Wang et al. (2009) have used a Soltan-type argument (Soltan 1982) to show that the average radiative efficiency of SMBHs has decreased over time, as might be expected if SMBHs grow predominately through episodic accretion events that act to lower their spin. Using cosmological simulations which followed the buildup of large SMBHs at $z=6$, Sijacki et al. (2009) showed that black hole mergers can contribute to the spin-down of SMBHs as well, resulting in low radiative efficiencies and rapid growth. A decrease in radiative efficiency must be reconciled with observations of an increase in the fraction of radio-loud quasars with decreasing redshift (Jiang et al. 2007), which would suggest SMBHs with larger spins (and hence larger efficiencies) at low- $z$. Therefore, understanding the behavior of angular momentum in the circumnuclear region is an important part of modeling accretion, and subsequently AGN feedback.

Simulations that follow the growth of SMBHs over cosmological times, or over the duration of a galaxy merger, often cannot follow the circumnuclear regions of galaxies with high enough resolution to describe the accretion flow in detail. Such simulations must make approximations for the accretion rates using the properties of the galaxies on scales that are resolved. A common technique is to assume that the unresolved disk is fed by Bonditype accretion (Hoyle \& Lyttleton 1939; Bondi \& Hoyle 1944; Bondi 1952), as in for example the smoothed particle hydrodynamics simulations of Springel et al. (2005a) who estimate the accretion rate based on the properties of the gas on a scale of $\sim 100 \mathrm{pc}$. The assumption of Bondi accretion appears to be reasonable for following evolution over cosmological times, where the average accretion rate onto the black hole cannot be more than the average accretion rate through any radius, since the fuel supply is limited by large scales. Previous cosmological simulations have been successful in reproducing observed population demographics and trends, such as the black hole mass function and galaxy colors and morphologies (as in, e.g., Di Matteo et al. 2008; Croft et al. 2009; McCarthy et al. 2009). However, for detailed studies of the growth and evolution of individual SMBHs or of the coupling between AGN feedback and the black hole accretion rate, more accurate descriptions of the accretion rate and its dependence on the small-scale features of the host galaxy are desirable.

Small-scale simulations have addressed gas dynamics in subgalactic-scale disks with high resolution (Fukuda et al.|2000; Wada|2001; Wada \& Norman 2001; Escala 2007; Wada \& Norman 2007; Kawakatu \& Wada 2008), finding the development of a turbulent, multiphase interstellar medium. The approximation of Bondi accretion in such an environment is not necessarily invalid. Krumholz et al. (2005) have shown that modified forms of the Bondi prescription can describe accretion in turbulent environments. A simulation must be equipped to model the properties of the turbulence in order to employ the modified Bondi prescription. If the gas contains a significant amount of angular momentum, the unmodified Bondi prescription gives inaccurate estimates of the accretion rate as well (Proga \& Begelman 2003; Krumholz et al. 2006). However, even modified Bondi prescriptions become inapplicable in the case of the selfgravitating, rotationally supported disks that are likely to form as large amounts of gas are driven inward in high-redshift galaxies. As the use of adaptive techniques increasingly improves the resolution of cosmological simulations, accretion onto black holes can no longer be described by approximate prescriptions such as Bondi accretion.

In the present paper, we use cosmological adaptive mesh refinement simulations with a large dynamic range to study the transport of gas and angular momentum through the circumnuclear disk of an SMBH host galaxy over time. The goal is to provide a description of accretion that can be compared to prescriptions typically applied in larger scale simulations. It will be shown that in the limiting case of relative quiescence (e.g., in between merger events and without AGN feedback) accretion is a stochastic rather than a continuous process. The method behind the simulations used here is described in detail in Levine et al. (2008), hereafter Paper I, and in Levine (2008). In Section 2, we briefly summarize the details of the simulations. The results of the simulations are given in Sections 3,5, including an analysis of the mass accretion rate and the angular momentum of the gas in the circumnuclear region of the galaxy. Finally, we summarize and discuss the results in Section 6 .

\section{SIMULATION}

The simulations presented here were run using the Adaptive Refinement Tree (ART) code (Kravtsov et al. 1997; Kravtsov 1999; Kravtsov et al. 2002), following the "zoom-in" method described in detail in Paper I. The code follows gas hydrodynamics on an adaptive mesh and includes dark matter and stellar particles. The gas cooling and heating rates are tabulated as functions of density, temperature, metallicity, and redshift over the temperature range $10^{2}<T<10^{9} \mathrm{~K}$ using CLOUDY (Ferland et al. 1998), which accounts for the metallicity of the gas, the formation of molecular hydrogen and cosmic dust, and UV heating due to cosmological ionizing background. Because the circumnuclear disk of the simulated galaxy reaches high densities, the gas can become optically thick to its own cooling radiation. In order to account for this effect and approximate optically thick cooling, we have also run a simulation that uses a Sobolev-like approximation for the column density of the gas to account for the large opacity of the highest-density regions in the simulated galaxy in calculating the cooling rates (see Gnedin et al. 2009).

Stellar particles are formed in cells with appropriate densities and temperatures at an efficiency that matches observed star formation rates on kiloparsec scales (Kennicutt 1998) and 100 pc scales (e.g., Young et al. 1996; Wong \& Blitz 2002). Presently, only the cosmological portion of the simulation (before zooming-in) includes radiative transfer and feedback and enrichment 
TABLE 1

Summary of Each Simulation Run

\begin{tabular}{|c|c|c|c|c|c|c|c|}
\hline Run & $\bar{z}$ & $\begin{array}{c}M_{200} \\
\left(10^{11} h^{-1} \mathrm{M}_{\odot}\right)\end{array}$ & $\begin{array}{c}M_{\mathrm{g}}^{\mathrm{a}} \\
\left(10^{10} h^{-1} \mathrm{M}_{\odot}\right)\end{array}$ & $\begin{array}{c}M_{*} \\
\left(10^{9} h^{-1} \mathrm{M}_{\odot}\right)\end{array}$ & $\begin{array}{c}M_{\bullet} \\
\left(10^{7} \mathrm{M}_{\odot}\right)\end{array}$ & $\begin{array}{c}\Delta x_{\min } \\
\text { (proper pc) }\end{array}$ & $\begin{array}{c}\text { Duration } \\
\left(10^{6} \mathrm{yr}\right)\end{array}$ \\
\hline Z3L20 & 3 & 2.4 & 3.3 & 7.3 & 3 & 0.032 & 0.84 \\
\hline Z3.5L20 & 3.5 & 2.0 & 2.8 & 5.3 & 3 & 0.028 & 0.71 \\
\hline Z4L15 & 4 & 1.7 & 2.5 & 4.0 & 3 & 0.819 & 1.0 \\
\hline Z4L18 & 4 & 1.7 & 2.5 & 4.0 & 3 & 0.102 & 1.0 \\
\hline $\mathrm{Z} 4 \mathrm{~L} 20^{\mathrm{b}}$ & 4 & 1.7 & 2.5 & 4.0 & 3 & 0.026 & 1.9 \\
\hline Z4L20.B3 & 4 & 1.7 & 2.5 & 4.0 & 9 & 0.026 & 1.0 \\
\hline Z4L20.B10 & 4 & 1.7 & 2.5 & 4.0 & 30 & 0.026 & 0.80 \\
\hline $\mathrm{Z} 4 \mathrm{~L} 20 . \mathrm{OT}^{\mathrm{c}}$ & 4 & 1.7 & 2.5 & 4.0 & 3 & 0.026 & 0.85 \\
\hline Z6L20 & 6 & 0.66 & 1.0 & 0.84 & 3 & 0.018 & 1.9 \\
\hline
\end{tabular}

a Mass of gas with $T<20,000 \mathrm{~K}$.

b Fiducial run.

c Includes a Sobolev-like approximation for the column density to model optically thick cooling.

from stars (although stellar particles are still formed in the zoom-in simulations). The physics on small scales in galaxies is complex, so our approach is to individually address each problem, carefully building a realistic, state-of-the-art simulation one piece at a time.

Here, we provide a brief overview of the zoom-in method, with a complete description given in Paper I. A cosmological simulation is evolved from a random realization of a Gaussian density field at $z=50$ in a periodic box of $6 h^{-1} \mathrm{Mpc}$ with an appropriate power spectrum, and is followed assuming a flat $\Lambda \mathrm{CDM}$ model: $\Omega_{0}=1-\Omega_{\Lambda}=0.3, \Omega_{\mathrm{b}}=0.043, h=H_{0} / 100=0.7$, $n_{\mathrm{s}}=1$, and $\sigma_{8}=0.9$. Beginning with the cosmological simulation, which has a maximum resolution of $\approx 180 h^{-1} \mathrm{pc}\left(37 h^{-1} \mathrm{pc}\right.$ proper at $\left.z=4\right)$, the resolution is slowly increased one refinement level at a time, reaching a quasi-stationary state on each level before increasing to the next level. The final, maximum resolution in our fiducial run is $\approx 0.089 h^{-1}$ pc $\left(0.02 h^{-1}\right.$ pc proper at $z=4$ ), corresponding to 20 levels of refinement.

After reaching the maximum resolution, a fraction of the gas in the center of the galaxy is replaced with a black hole particle of equal mass and momentum. The circumnuclear region is extremely gas rich, with the gas comprising $91 \%, 83 \%$, and $79 \%$ of the baryon mass inside a radius of $1 \mathrm{kpc}$ at $z=6,4$, and 3 , respectively. In this region, the gas mass dominates over all other components, including the black hole particle. The black hole mass has not been chosen to match any of the local scaling relations (e.g., Magorrian et al. 1998; Ferrarese \& Merritt 2000; Gebhardt et al. 2000; Tremaine et al. 2002). Instead, we have chosen a mass that is large enough to distinguish it from stellar particles in the simulation, and that tends to stay put in the center of the galaxy, but does not depend on any a priori assumptions about how the scaling relations extend to high redshift. The possibility remains that the simulated galaxy should host a more massive black hole, but since we do not follow the black hole's growth from its seed formation, the mass of the black hole particle is somewhat arbitrarily chosen. Presently, the mass of the black hole particle does not change over the course of the zoom-in simulations. This simplification allows us to avoid making any assumptions about how the gas mass on the smallest scales of the simulations will be accreted onto the black hole particle.

After the introduction of the black hole particle, the simulation continues to evolve with the maximum resolution for several hundred thousand years. The duration of each simulation varies with redshift. At lower redshifts, the increased mass of the galaxy results in more high-density cells, requiring high resolution. Following a larger portion of the simulation with finer resolution is more computationally expensive, therefore the lower redshift simulations take longer to evolve. As a result, e.g., the $z=3$ zoom-in is evolved only half as long in physical time as the fiducial $z=4$ simulation.

Most of the following analysis is applied to the shortterm evolution of the galaxy $\left(\sim 10^{6} \mathrm{yr}\right)$ during a zoom-in episode at $z=4$ containing a $3 \times 10^{7} \mathrm{M}_{\odot}$ SMBH particle (considered the fiducial run7). Additional simulations were performed, including zoom-in episodes at other redshifts with the same black hole mass $(z=3,3.5$, and $6)$, runs with different black hole masses $\left(9 \times 10^{7}\right.$ and $\left.3 \times 10^{8} \mathrm{M}_{\odot}\right)$ at $z=4$, two lower resolution versions of the fiducial run (with maximum levels of refinement 15 and 18), and a version of the fiducial run that implements an approximation for optically thick cooling. The $z=4$ simulation was chosen for the fiducial run because this redshift corresponds to a relatively quiescent stage in the growth of the host galaxy. At this stage, the main galaxy of the simulation has not undergone a large mass ratio merger in $\approx 400 \mathrm{Myr}$, although the galaxy is still actively growing. At $z=6$ (also studied here), the galaxy is less relaxed, as it is entering into a 4:1 merger at that time. It is expected that the mass of the black hole should continue to grow with decreasing redshift. Since the different redshift simulations each contain the same black hole particle mass, they do not provide the growth history of a single galaxy, but rather they represent the distinct growth histories of similar disk galaxies. Table 1 summarizes the details of the simulations performed, including redshift, the total mass within a radius enclosing a mean density of 200 times the present-day critical density of the universe, centered on the main galaxy $\left(M_{200}\right)$, the mass of gas with temperature less than $20,000 \mathrm{~K}\left(M_{\mathrm{g}}\right)$, the stellar mass $\left(M_{*}\right)$, the black hole mass $\left(M_{\bullet}\right)$, the size of the most refined cell in each run $\left(\Delta x_{\min }\right)$, and the duration of each run in physical time.

7 The fiducial run, Z4L20, is effectively the same simulation described in Paper I. The only difference is in the initial zoom-in of the run presented here, which occurred more slowly (see discussion in Section 2.2, Paper I). 


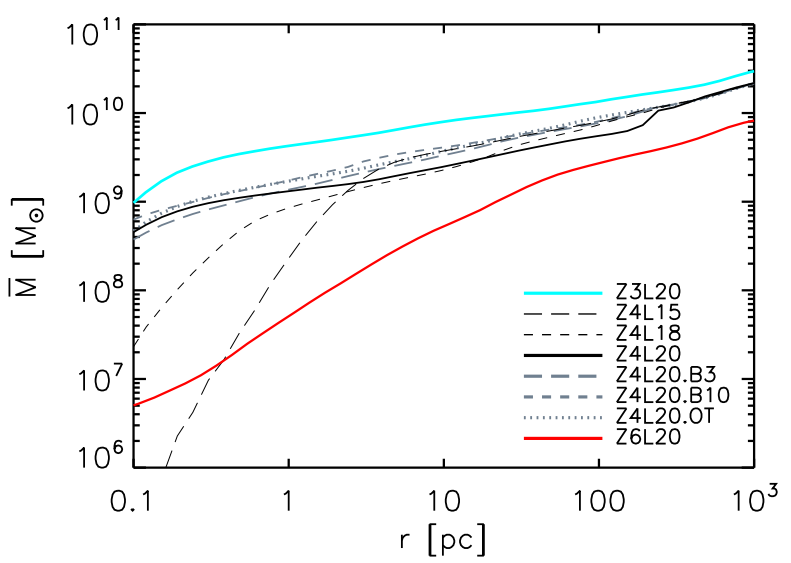

FIG. 1. - Time-averaged interior gas mass as a function of radius for each simulation.

Paper I gives the details of the spatial structure of the circumnuclear region for the fiducial run. Each run described here develops a similar structure: a cold, rotationally supported, self-gravitating gas disk develops in the circumnuclear region of the galaxy (inside $\sim 100 \mathrm{pc}$ ), with a steep power-law density distribution. The disk is globally unstable, leading to the development of waves and instabilities, which drive turbulence on a range of scales, injecting energy through shocks. The turbulence is highly supersonic, effectively raising $Q$ and acting to maintain local stability in the disk. In the run Z4L20.OT, the approximation for optically thick cooling accounts for the densest gas being optically thick to its own cooling radiation. Consequently, the mass-weighted mean temperature in that run is higher than in the fiducial run, which contributes to a slightly higher Toomre $Q$ parameter. In either case, the higher effective $Q$ prevents the circumnuclear disk from fragmenting entirely into star-forming clumps on a free-fall time. However, the actual star formation rate in the simulation does not reflect the complicated behavior of the circumnuclear region, because the rate is simply density- and temperaturedependent and operates on timescales much longer than the million-year time scales of the zoom-in simulation. During the zoom-in simulation, the disk shows transient features caused by spiral waves and global instabilities, which slowly allow angular momentum transport, driving gas inward toward the center of the galaxy. Similar behavior, where global instabilities generate stabilizing turbulence, has been observed in other numerical simulations (Regan \& Haehnelt 2009; Wise et al. 2008; Englmaier \& Shlosman 2004) and has been studied analytically by Begelman \& Shlosman (2009). In the following sections, we explore the behavior over time of the mass accretion rate and the angular momentum of the gas throughout the circumnuclear disk.

\section{DISK MASS AND THE RATE OF TRANSPORT}

Figure 1 shows the mean gas mass, $\bar{M}$ (averaged over the duration of the simulation), interior to radius, $r$, for each simulation (except $z=3.5$ ). The radius $r$ is measured with respect to the position of the black hole particle, which approximates the center of the galaxy over the duration of each zoom-in simulation (except for a few brief intervals, which are discussed later in this section).
A comparison of the average gas mass in each zoom-in episode approximately shows the growth of the circumnuclear disk over cosmological times. The substantial growth in mass between $z=6$ and $z=4$ is caused by a major merger (with a mass ratio of 4:1) and a few minor mergers occurring in between.

In contrast with the averaged mass-profiles, the instantaneous mass-profiles at individual times during the zoom-in part of the simulation (not shown) reveal the transient nature of the circumnuclear disk. As the globally unstable disk forms transient structures on a range of scales, the interior gas mass can vary significantly. Figure 2 shows the amplitude of mass fluctuations, $M(t) / \bar{M}$ at four different radii (ranging from 0.1 to $100 \mathrm{pc}$, spanning scales which have undergone at least one rotation period over the course of each of the zoom-in simulations) over time for each of the three redshift simulations. The figure gives an indication of the turbulent nature of the gas in the circumnuclear disk of the galaxy. In their high-resolution re-simulations of mergers between disk galaxies, Hopkins \& Quataert (2009) find a qualitatively similar picture, in which global instabilities operate on a range of scales, causing a great deal of fluctuation in the interior gas mass as a function of time throughout the circumnuclear disk.

Around 550,000 yr in the $z=4$ simulation, a $\sim$ few $\times 10^{7} \mathrm{M}_{\odot}$ clump of gas (which forms in the central $10 \mathrm{pc}$, perhaps as a result of the global instabilities in the disk) moves within a few parsecs of the black hole particle, temporarily creating a gravitational disturbance that displaces the black hole particle for $\approx 50,000 \mathrm{yr}$. The black hole soon resettles into the bottom of the potential well of the disk, but because the interior mass is measured with respect to the black hole particle, the interior gas mass measurements are temporarily affected by the black hole's motion (see the dotted lines in Figure 2). Therefore, the interior gas mass shown in Figure 2 has been interpolated over the duration of the black hole displacement in all subsequent computations (unless otherwise noted). The properties of the clump of gas may be significant for the angular momentum of the black hole accretion disk, which will be discussed further in Section 5. Runs Z4L20.BH3, Z4L20.BH10, and Z4L20.OT undergo similar events of variable duration. The details of each displacement, such as the size and the duration of the subsequent relaxation, depend on the somewhat arbitrarily chosen black hole particle mass and the gas dynamics, which vary between different runs. Therefore, even though the effect of in-falling clumps on the gas disk may be significant, the displacement of the black hole particle in these simulations does not necessarily imply that such displacements occur similarly in nature.

As Figure 2 shows, a characteristic accretion rate is not straightforwardly determined by any one individual snapshot of the circumnuclear region, given the amount of fluctuation in the gas mass over the timescale of the simulations. A simple estimate of the net accretion rate could be obtained by measuring the difference in mass at each scale between the beginning and the end of each run. However, the dynamical and turbulent behavior of the circumnuclear disk gives rise to a highly variable accretion rate, often indicating as much outflow as inflow of gas mass, and in some instances, resulting in a net 


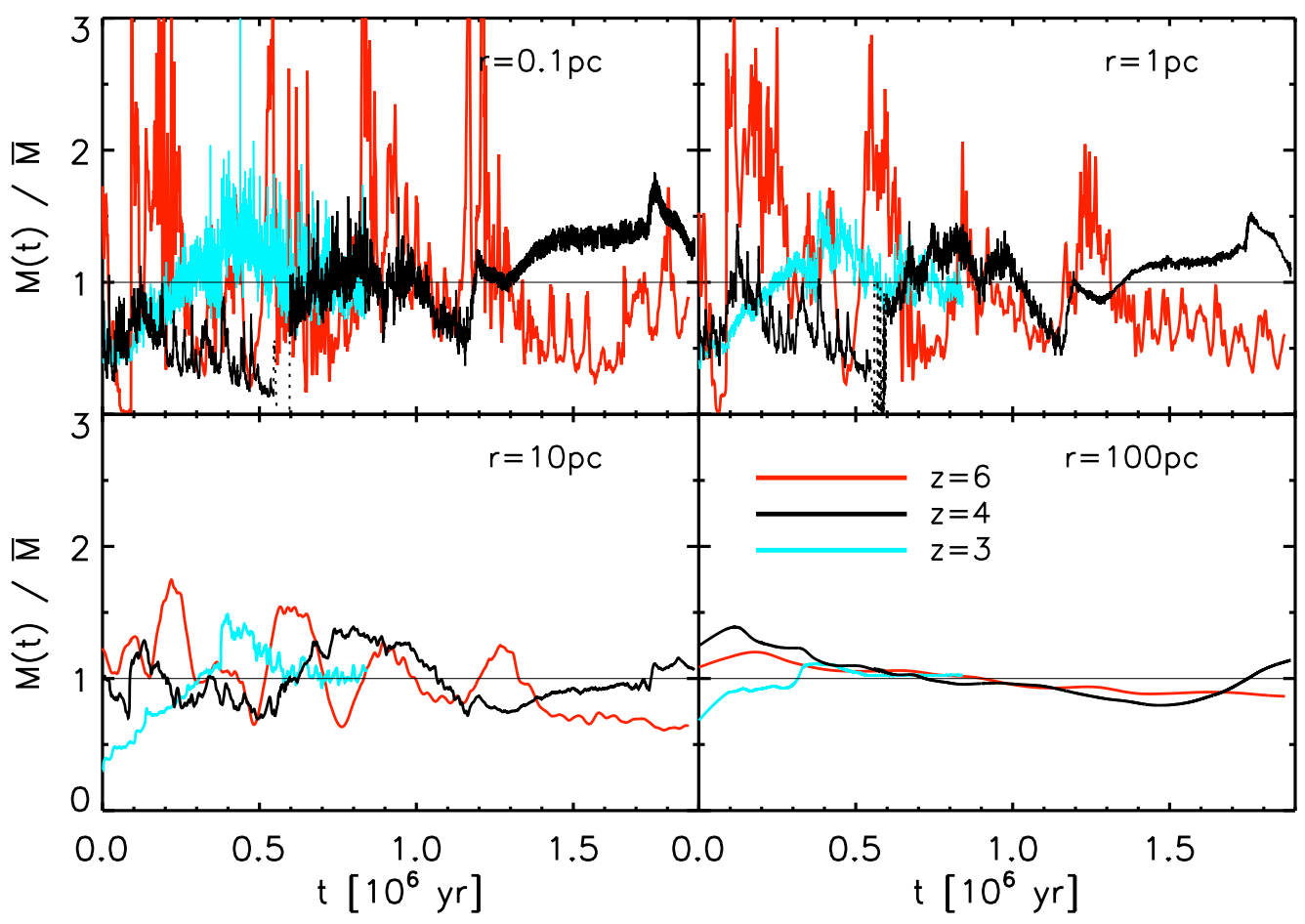

FIG. 2.- Amplitude of gas mass fluctuations at redshifts 3, 4, and 6 for four different radii. The dotted black line shows the fluctuations measured with respect to the black hole particle position over the duration of the black hole displacement. The thin solid line shows $M=\bar{M}$ for comparison. (Figure 1 shows $\bar{M}$ as a function of radius, $r$, for each simulation).

outflow. In this case, a Fourier transform of the interior gas mass provides a natural characterization of the mass fluctuations and their dependence (if any) on timescale.

We have chosen the sampling frequency for the mass (and all other physical quantities), somewhat arbitrarily, to correspond to one time step on level 6 . The frequency is such that the entire zoom-in region in each of the simulations steps at least 8 times (corresponding to the least-resolved level of refinement in the circumnuclear disk; level 9), and at most 16,384 times (corresponding to the most-resolved level; level 20)8. During the simulation, the physical time corresponding to each step is redetermined after every level-0 step, and depends on the dynamics of the disk (so that the time step obeys a global CFL condition). Therefore, the length of time between samplings varies throughout the duration of the simulation. The Lomb-normalized periodogram (see Lomb 1976; Press et al. 1992, Numerical Recipes, Section 13.8), or the spectral power normalized by the variance of a data set, is useful for studying the behavior of unevenly sampled data, such as the uneven time steps here. The normalized spectral power as a function of the angular frequency, $\omega$, is given by

\footnotetext{
8 Time steps forward in the simulation so that for each step on level 6 , the simulation has taken $2^{l-6}$ steps on level $l$.
}

$$
\begin{aligned}
P_{\mathrm{N}}(\omega) & =\frac{\left[\sum_{j}\left(M_{j}-\bar{M}\right) \cos \omega\left(t_{j}-\tau\right)\right]^{2}}{2 \sigma_{\mathrm{M}}^{2} \sum_{j} \cos ^{2} \omega\left(t_{j}-\tau\right)} \\
& +\frac{\left[\sum_{j}\left(M_{j}-\bar{M}\right) \sin \omega\left(t_{j}-\tau\right)\right]^{2}}{2 \sigma_{\mathrm{M}}^{2} \sum_{j} \sin ^{2} \omega\left(t_{j}-\tau\right)},
\end{aligned}
$$

summed over $N$ data points, where $\sigma_{\mathrm{M}}^{2}$ is the variance in the interior gas mass. The quantity $\tau$, defined by

$$
\tan (2 \omega \tau)=\frac{\sum_{j=1}^{N} \sin 2 \omega t_{j}}{\sum_{j=1}^{N} \cos 2 \omega t_{j}}
$$

is introduced so the spectral power is independent of shifting the data in time. Although the Lomb-normalized periodogram is not directly computed from the Fourier transform of the data, we use it to estimate the Fourier transform of the interior gas mass as follows: 


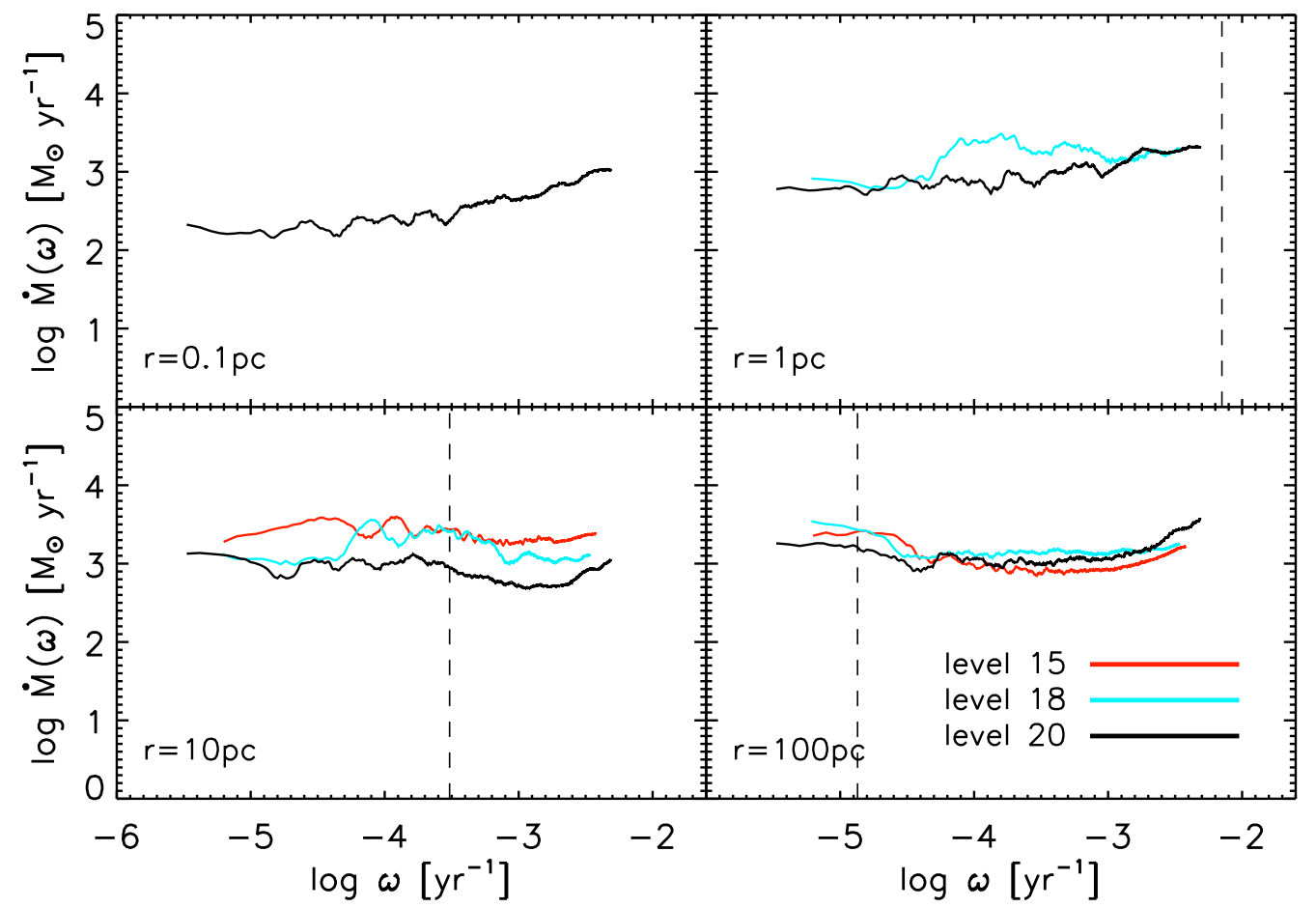

FIG. 3.- Mass flux, estimated from the spectral power of the interior gas mass fluctuations, for three different maximum resolution simulations $(15,18$, and 20 levels of refinement) at $z=4$. Here, the flux is only calculated for the first million years of run Z4L20, for a consistent comparison with runs Z4L15 and Z4L18.

$$
\begin{aligned}
M(\omega) & =\frac{1}{N} \sum_{j=1}^{N} M\left(t_{j}\right) e^{i \omega t_{j}} \\
& \approx \sqrt{\frac{P_{\mathrm{N}} \sigma_{\mathrm{M}}^{2}}{N}} .
\end{aligned}
$$

The Fourier transform of the mass follows a power law with slope $\approx-1$ in each of the simulations, showing no substantial departure down to frequencies comparable to the rotational period of the disk. The time derivative of the mass Fourier transform gives a characteristic "accretion rate" through radius $r$ as a function of frequency,

$$
\dot{M}(\omega)=i \omega M(\omega)
$$

Strictly speaking, the accretion rate given by Equation 4 does not describe the net inward transport of gas, but rather it gives typical values of the flux of gas mass into and out of a given region on a timescale $2 \pi / \omega$. The absolute value of the flux given by Equation 4 is shown as a function of frequency in Figures 3,5. The data are smoothed over neighboring points (the number of neighbors used in the smoothing is proportional to angular frequency, $\omega)$ to reduce the noise so that the different curves are readily distinguishable. Figure 3 shows a comparison of the $z=4$ runs with different maximum resolution (Z4L15, Z4L18, and Z4L20). The mass flux in each run is only shown for scales that are resolved by four cells or more. We find good agreement in the behavior shown for different resolution simulations, indicating that the results are converged with respect to resolution.
The mass flux is larger for decreasing redshift, because the galaxy itself is more massive, but the flux follows the same stochastic behavior at different epochs (Figure4), as well as for different black hole masses and for the optically thick cooling run (Figure 5). We note that $0.1 \mathrm{pc}$ (proper) is the resolution limit at $z=4$ (corresponding to four level-20 cells), and therefore this scale is barely resolved at $z=4$ and under-resolved at $z=3$. Therefore, the top-left panel of Figure 4 does not show the $z=3$ simulation. The vertical lines in each panel correspond to the average angular speed of the gas at a given scale in each simulation. The fluctuations do not appear to be correlated with the orbital period at any radius in the circumnuclear disk. The flat slope in the flux, $\dot{M}(\omega)$, shows that the rate of transport is independent of frequency, i.e., that accretion is a stochastic process with no preferred timescale. The result is generic over each run studied here, including the optically thick cooling run, which is dynamically similar to the other runs despite the presence of higher temperature gas throughout the circumnuclear disk. Simulations of self-gravitating disks show behavior similar to our own results, with instabilities driving turbulence (Wada \& Norman 1999; Wada 2001; Wada et al. 2002), which ultimately leads to intermittent fueling episodes varying on timescales of $10^{4}-10^{5}$ yr (Fukuda et al. 2000; Wada 2004).

\section{COMPARISON TO OTHER ACCRETION PRESCRIPTIONS}

As mentioned in the Introduction, simulations that do not resolve the accretion disk of an $\mathrm{SMBH}$, or the region where the gravity of the SMBH dominates, typically 


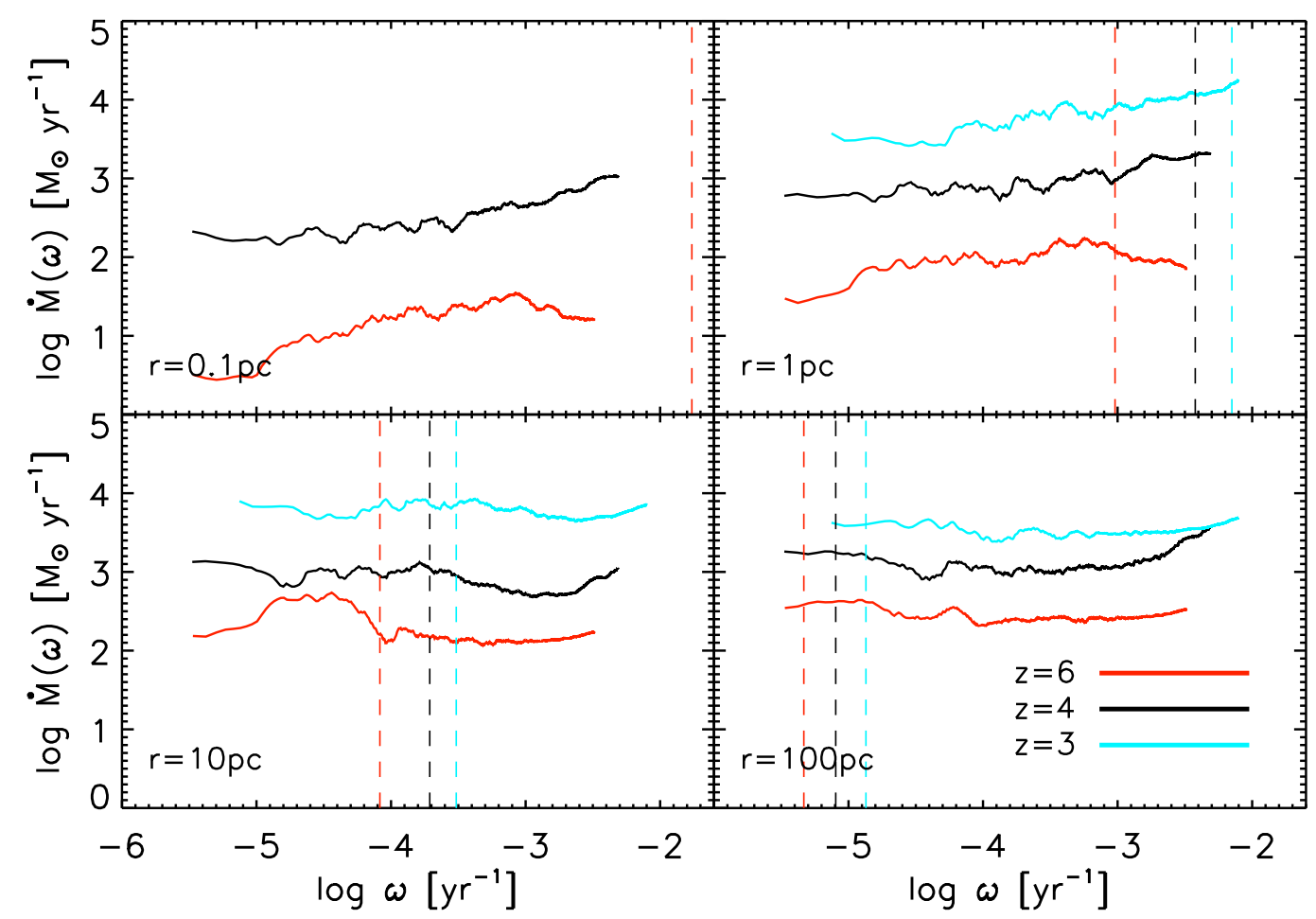

Fig. 4.- Mass flux, estimated from the spectral power of the interior gas mass fluctuations, for three different redshifts. The vertical lines correspond to the mean angular speed of the gas disk at radius, $r$, in each simulation.

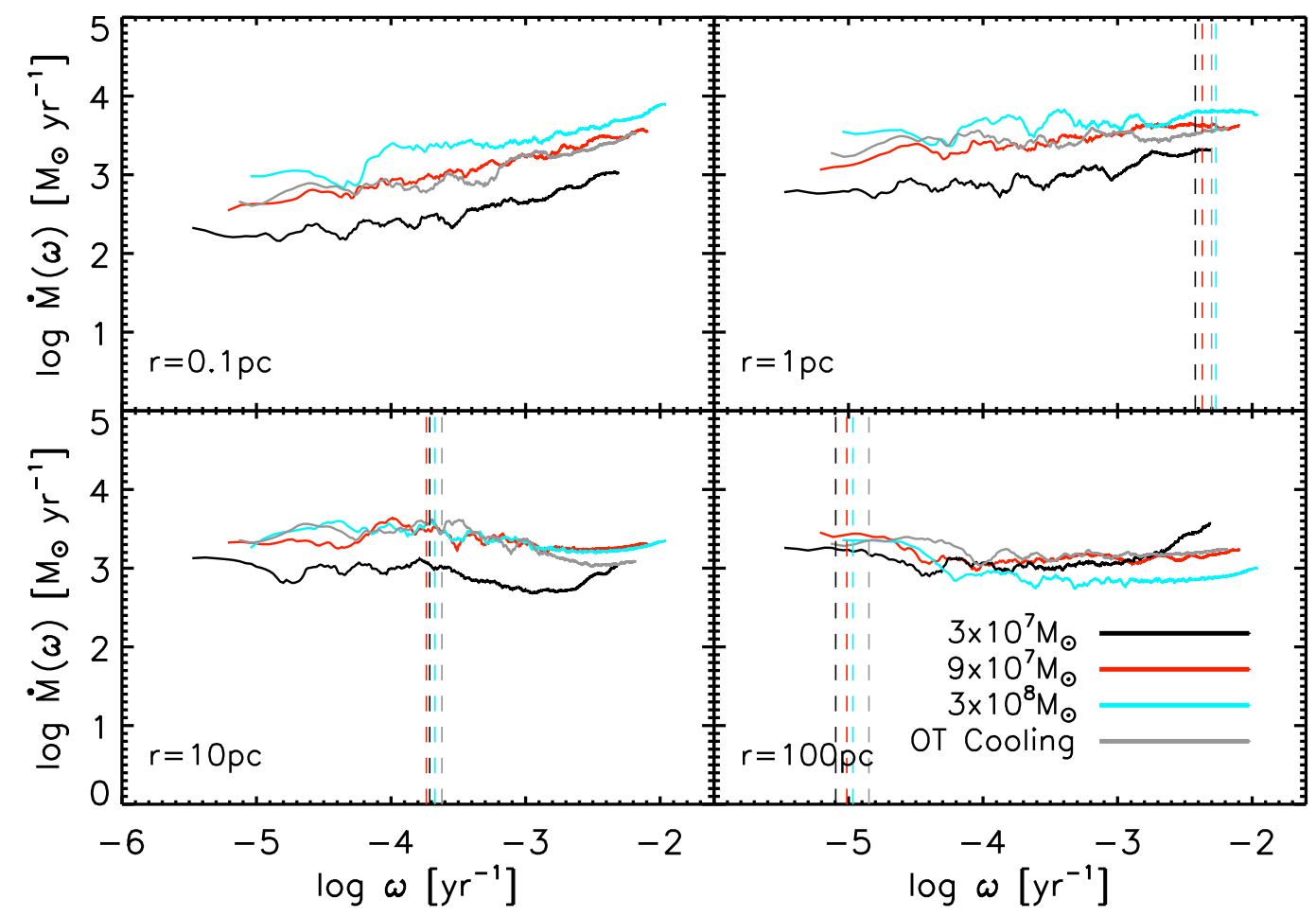

Fig. 5.- Mass flux, estimated from the spectral power of the interior gas mass fluctuations, for three different black hole masses and for the optically thick cooling run at $z=4$. The vertical lines correspond to the mean angular speed of the gas disk at radius, $r$, in each simulation. 
include some prescription for the accretion rate that depends on the properties of the simulated galaxy on scales that are resolved. It is useful to check how well a prescription, such as Bondi accretion, describes mass transport when extended down to parsec scale resolution.

The original Bondi prescription assumes pressuresupported gas in hydrodynamic equilibrium. Turbulence, as well as angular momentum (such as in a disk), has been shown to lower the actual accretion rate below the Bondi rate (Abramowicz \& Zurek 1981; Proga \& Begelman 2003; Krumholz et al. 2006). The circumnuclear disk in the simulations here is both turbulent and rotationally supported (see Figure 6 of Paper I), bearing little resemblance to the medium modeled in the original Bondi prescription. Modifications to the Bondi prescription that account for both turbulence and vorticity in gas can be applied to estimate the reduced accretion rate (Krumholz et al. 2005, 2006). However, even such a modified prescription fails to describe accretion of self-gravitating gas, such as in the circumnuclear disk of the present simulations. Disks that are self-gravitating form bars and spiral waves that further change the flow of gas.

Figure 6 shows a comparison of the Bondi Hoyle rate for turbulent gas (computed using the spherically averaged radial profiles of the gas density, turbulent velocity, and sound speed) and the vorticity-reduced rate, applied to each redshift simulation. In calculating the Bondi Hoyle rate for turbulent gas, $\dot{M}_{\mathrm{BH}}$, the thermal sound speed of the gas has been replaced by an effective sound speed defined as the quadrature sum of the thermal sound speed and turbulent velocity. The vorticity-reduced rate, $\dot{M}_{\text {turb }}$, given by Equation 3 of Krumholz et al. (2006), is computed cell by cell, producing a log-normal probability density function (PDF) of accretion rates within individual spherical shells centered on the black hole particle. The geometric mean of the distribution, $\left\langle\dot{M}_{\text {turb }}\right\rangle$, gives characteristic accretion rates under this prescription and is shown for several different radii in Figure 6. The vorticity-reduced rates are $2-3$ orders of magnitude smaller than the standard Bondi Hoyle rates. These results are qualitatively consistent with those of DeBuhr et al. (2009), who find that accretion in their merger simulations is regulated by angular momentum transport processes in the host galaxy, keeping the accretion rate below the Bondi rate, except during peak activity (when the accretion rate approaches $\dot{M}_{\text {Edd }}$, which is $\left.\approx \dot{M}_{\mathrm{BH}}\right)$.

As revealed in Figure 6, the Bondi prescription gives large estimates for the accretion rates $\left(\gg 10 \dot{M}_{\text {Edd }}\right)$ inside the central few hundred parsecs. The steep, power-law, density profile of the gas in the circumnuclear disk contributes to the large accretion rates shown in Figure 6 (which are proportional to density). The inclusion of an approximation for optically thick cooling in the simulations does not significantly change the density profile or the velocity dispersion of the gas. Therefore, the Bondi prescription produces similar results for runs Z4L20 and Z4L20.OT (dashed and solid curves in Figure 6). Additional effects not included in our current simulations may contribute to the depletion of gas in the circumnuclear disk, such as AGN feedback and stellar feedback (not included in the zoom-in portion of the simulation),

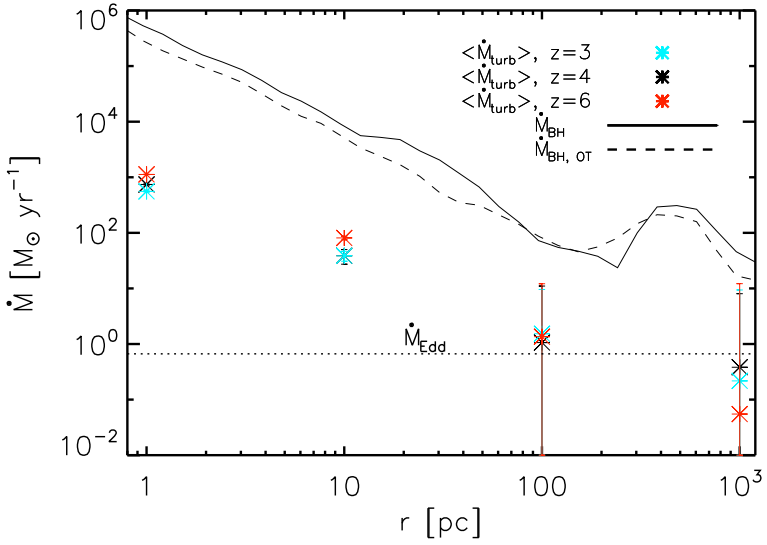

FIG. 6. - Bondi accretion rate as a function of radius for different redshifts. Two methods of estimation are shown: (1) $\dot{M}_{\mathrm{BH}}$ computed from spherically averaged quantities (solid black curve: fiducial run, dashed curve: optically thick cooling run) and (2) the geometric mean and standard deviation of the $\dot{M}_{\text {turb }}$ PDF (points). The Eddington accretion rate, $\dot{M}_{\text {Edd }}$ for a 30 million $\mathrm{M}_{\odot}$ black hole with a radiative efficiency of 0.1 is also shown for comparison (dotted black line).

thus lowering the estimated Bondi rate. However, if the disk remains self-gravitating and therefore susceptible to instabilities, the Bondi prescription will inaccurately describe the transport of gas through this region.

The average accretion rate through the circumnuclear disk over cosmological times can be estimated by comparing the mean interior gas mass (the averages shown in Figure 1) for the different redshift simulations. Figure 7 shows the mean gas mass, interior to radius $r$ as a function of the age of the universe, $t_{\text {age }}$. Once again we emphasize that the different redshift simulations do not necessarily describe different stages of growth of the same galaxy because they each contain the same mass SMBH (rather than a black hole that grows with redshift). However, the black hole particle does not currently play a large role in the evolution of the simulated galaxy (at least not below $z=4$, where the mass of the black hole is dominated by the gas mass all the way down to the resolution limit).

The dashed line in Figure 7 shows the mass of a black hole, initially $3 \times 10^{7} \mathrm{M}_{\odot}$ at $z=6$, if it grows continuously at the Eddington limit according to

$$
M(t)=M_{\bullet} \mathrm{e}^{t / t_{\mathrm{S}}}
$$

where $t_{\mathrm{S}}$ is the Salpeter time (Salpeter 1964) of $4.5 \times$ $10^{7} \mathrm{yr}$, for a radiative efficiency $\eta=0.1$. If the dynamics of the circumnuclear disk extend all the way down to scales beneath the resolution (which cannot be assumed) then the growth rate approximated by the simulation points, which appears to steepen at early times with decreasing scale, may continue to steepen down to smaller scales, approaching the Eddington limit. Therefore, a black hole in our simulations will be able to grow according to Equation 5 at high- $z$, as long as efficient fueling from the circumnuclear disk can be sustained. This is perhaps consistent with the isolated disk galaxy simulations of Springel et al. (2005a), where a sufficiently massive black hole will grow according to Equation [5] in the 


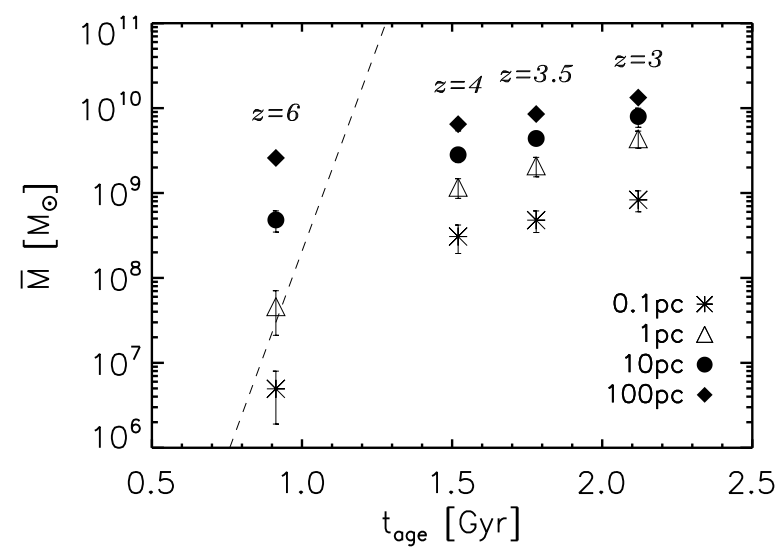

FIG. 7.- Mean gas mass interior to radius $r$ as a function of the age of the universe, $t_{\text {age }}$. The error bars correspond to the dispersion in the interior gas mass over each run. A $z=3.5$ simulation, not mentioned above, is also included in the figure.

absence of AGN feedback. Once gas reaches sub-parsec scales, the actual accretion rate onto the black hole is governed by the physics of the accretion disk, not modeled by our simulations. Nonetheless, Figure 7 shows that the increase in the mass of the circumnuclear gas disk over cosmological times yields enough fuel to build up the mass of the SMBH if the fuel can be efficiently accreted.

\section{ANGULAR MOMENTUM}

The large fluctuations in the interior gas mass correspond to waves moving through the circumnuclear disk, which can potentially drive individual clumps of molecular gas into the vicinity of the SMBH. The fluctuations develop stochastically, as shown in Section 3 , which may ultimately lead to stochastic SMBH accretion events. If the direction of the angular momentum vector of accreted material with respect to the black hole's spin axis also varies stochastically, some fraction of accretion events will be anti-aligned, lowering the black hole's spin and consequently its radiative efficiency (King \& Pringle 2006, 2007; Nayakshin \& King 2007; King et al. 2008). However, large amounts of accretion, as in merger driven fueling, will tend to align an SMBH's spin with that of the circumnuclear disk, increasing its radiative efficiency (Volonteri et al. 2007). Spin-down resulting from accretion could be more effective in non-merging disk galaxies hosting smaller SMBHs, as secular processes stochastically drive small amounts of gas toward the SMBH. The resulting low spin may be consistent with observations of disk galaxies as hosts for radio-quiet sources, if radioloudness is associated with the spin of the black hole (Berti \& Volonteri 2008). Since black hole mergers may also contribute to the spin-down of SMBHs (Sijacki et al. 2009), it is important to qualify the relative importance of gas accretion for determining black hole spin.

The simulations here do not model the gas down to scales inside the accretion disk, nor allow the black hole particle to accrete material from larger scales (as discussed in Section 2). For these reasons, the simulations do not follow the spin of the black hole. The simulations do model gas on parsec scales, enabling us to resolve the direction of the angular momentum vector of gas and to provide boundary conditions for simulations of $\mathrm{SMBH}$ accretion disks.

The angular momentum vector is measured with respect to the black hole particle's position and velocity. Some uncertainty in the direction of the angular momentum vector could arise because the position of the black hole does not necessarily coincide exactly with the position of the center of the galaxy and because the black hole may have a significant rotational velocity about the center. During most of the simulation, the black hole particle lies in the densest cell, which coincides with the minimum of the galaxy's gravitational potential. Therefore, the black hole particle typically provides a reasonable estimate of the location of center of the galaxy. We have also measured the angular momentum relative to a center whose velocity is defined by an average over the central cell and six of its neighbors, but find that the effect on the angular momentum vector is negligible (except for the brief periods of black hole particle displacement).

Figure 8 shows a map projection of the direction of the normalized angular momentum vector as it evolves in time at 100,10 , and $1 \mathrm{pc}$ from the black hole particle for the $z=4$ and $z=3$ simulations (left and right, respectively). The mean direction of the rotation axis of the galaxy on kiloparsec scales, which remains comparatively constant over the $\sim 1$ Myr duration of the zoom-in simulation, is oriented toward the center of the map in each case. The disk is slightly warped on scales smaller than a kiloparsec, so that the axis of the circumnuclear disk is oriented at an angle to the large-scale disk. Figure 9 shows the inclination angle of the angular momentum vectors shown in Figure 8 with respect to those measured at a kiloparsec, over time. Together, Figures 8 and 9 demonstrate the effect of the circumnuclear disk's chaotic behavior on the angular momentum of the accreting gas.

At $z=4$ and a scale of $100 \mathrm{pc}$, the direction of the angular momentum vector changes slowly but shows little scatter over the course of the simulation. There is a small amount of scatter during the displacement of the black hole particle, which is visible on each scale shown. Rather than interpolating the magnitude and direction of the angular momentum vector of the gas over the duration of the black hole displacement, as was done with the interior gas mass profile in Figure 2, we continue to measure quantities relative to the black hole particle. Once the black hole particle resettles into the bottom of the potential well, the excess scatter in $\boldsymbol{L} / L$ vanishes. The slow change in direction at $100 \mathrm{pc}$ may correspond to the increased warping of the disk as the simulation progresses. In a similar analysis of simulations of the growth of a disk galaxy by Saitoh \& Wada (2004), the spin axis of the central gas disk also changed direction on 100 pc scales (relative to kiloparsec scales).

The angular momentum vector at 10 and 1 pc starts out with the same approximate orientation as the larger scales but shows slightly more scatter. There are two sudden changes in the direction of the angular momentum vector at the $1 \mathrm{pc}$ scale. In the first incident, at $\sim 0.55 \mathrm{Myr}$, the angular momentum changes abruptly by more than $100^{\circ}$, coinciding with the time of the displacement of the black hole particle described in Section 


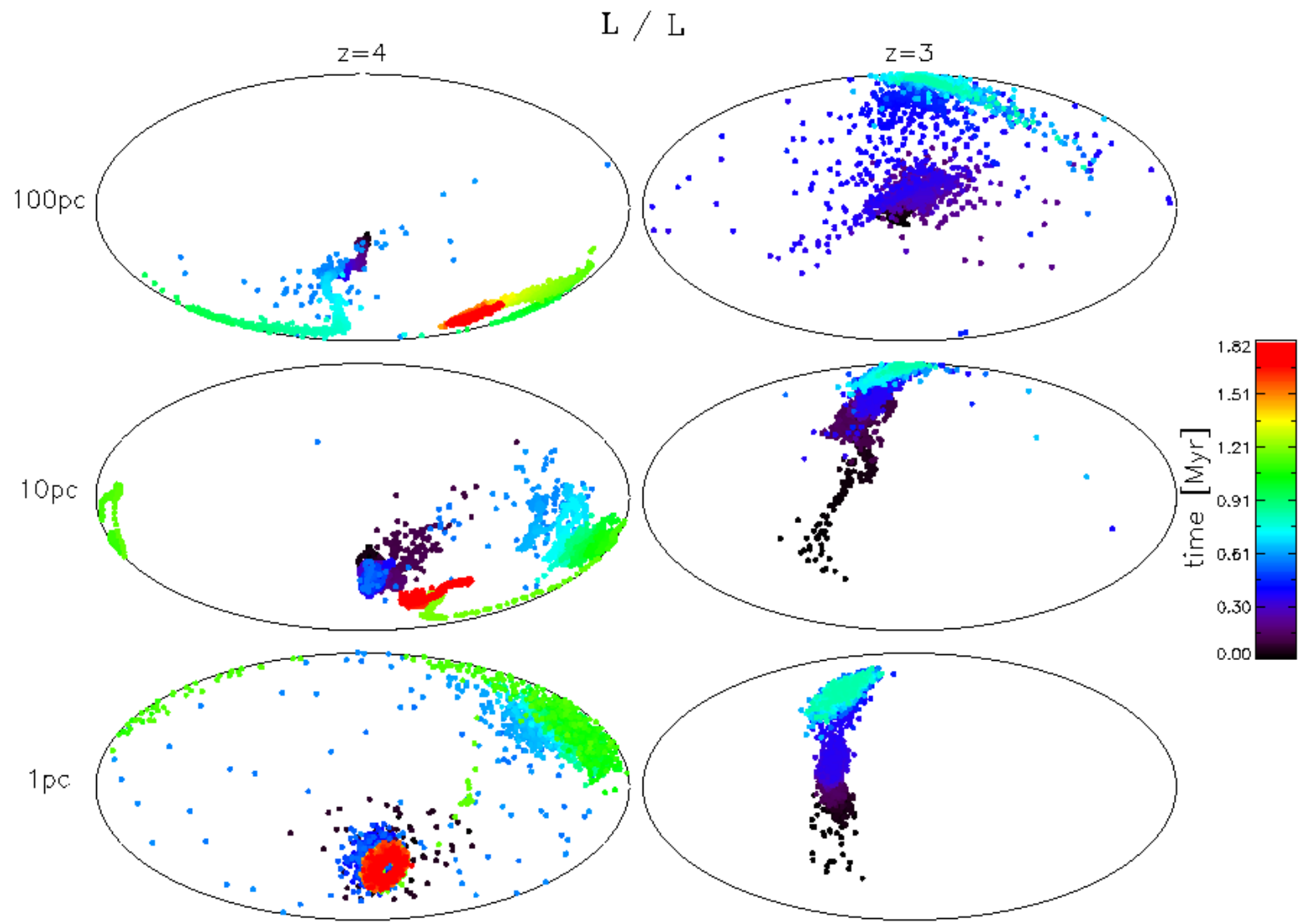

FIG. 8.- Map showing the direction of the normalized angular momentum vector, $\boldsymbol{L} / L$ as it changes over time at 100 , 10 , and $1 \mathrm{pc}$ in the $z=4$ and $z=3$ simulations. The colors correspond to the time elapsed in the simulation since the introduction of the black hole particle. Each map is aligned so that the mean rotation axis of the galactic disk at a scale of $1 \mathrm{kpc}$ lies in the center. This figure is best viewed in color.

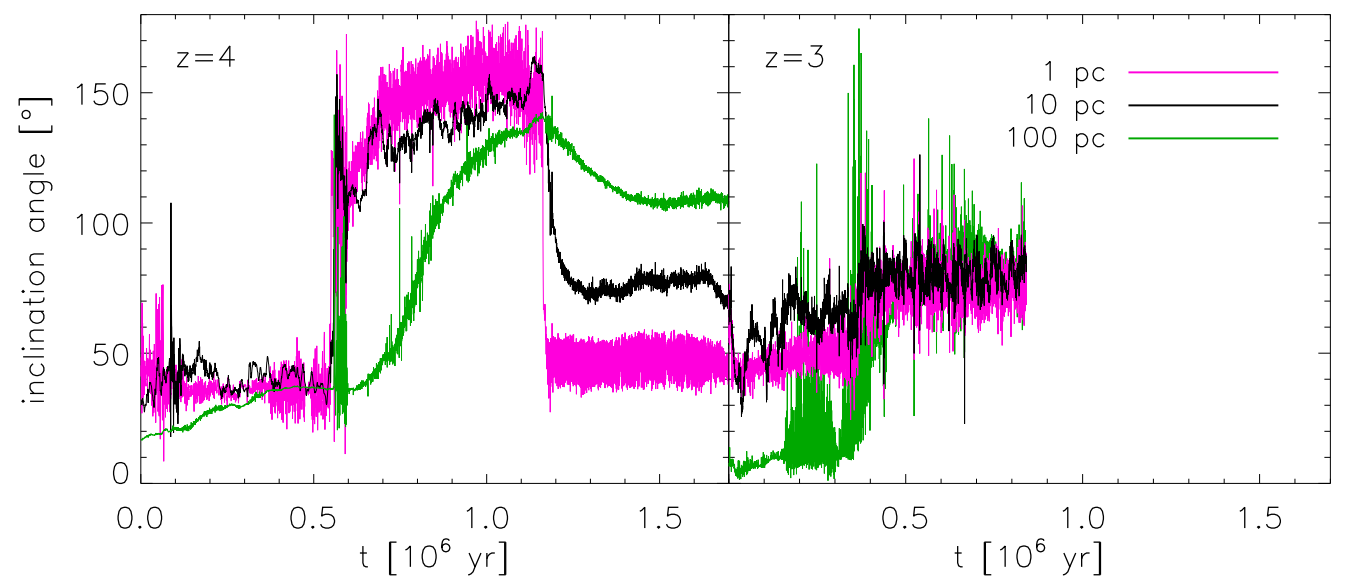

FIG. 9.- Inclination angle of the angular momentum of the inner disk with respect to that of the kiloparsec disk over physical time, for $z=4$ and $z=3$ (left and right panels, respectively).

3 (as a clump of gas forms near and moves into the center). The flip in direction is also visible at $10 \mathrm{pc}$. In the second incident, at $\sim 1.2 \mathrm{Myr}$, the angular momentum shifts by $\sim 100^{\circ}$ while the black hole remains stationary at the center of the galaxy. The second shift is the result of gravitational interaction with $\mathrm{a} \sim$ few $\times 10^{8} \mathrm{M}_{\odot}$ clump of gas, which develops at $>100 \mathrm{pc}$ and continues to move toward the center of the disk at late times. The mass of the clump is comparable to the mass of the disk interior to $\sim 10 \mathrm{pc}$. The second shift is also visible at the $10 \mathrm{pc}$ scale but occurs less suddenly. The two panels in Figure 10 show three-dimensional volume-renderings of the gas density at $30 \mathrm{pc}$ before and after the flip (top and bottom, respectively). The inset shows a zoomed-out view of the disk, where the large clump of gas is visible $\gtrsim 200 \mathrm{pc}$ from the center. If the clump remains intact 


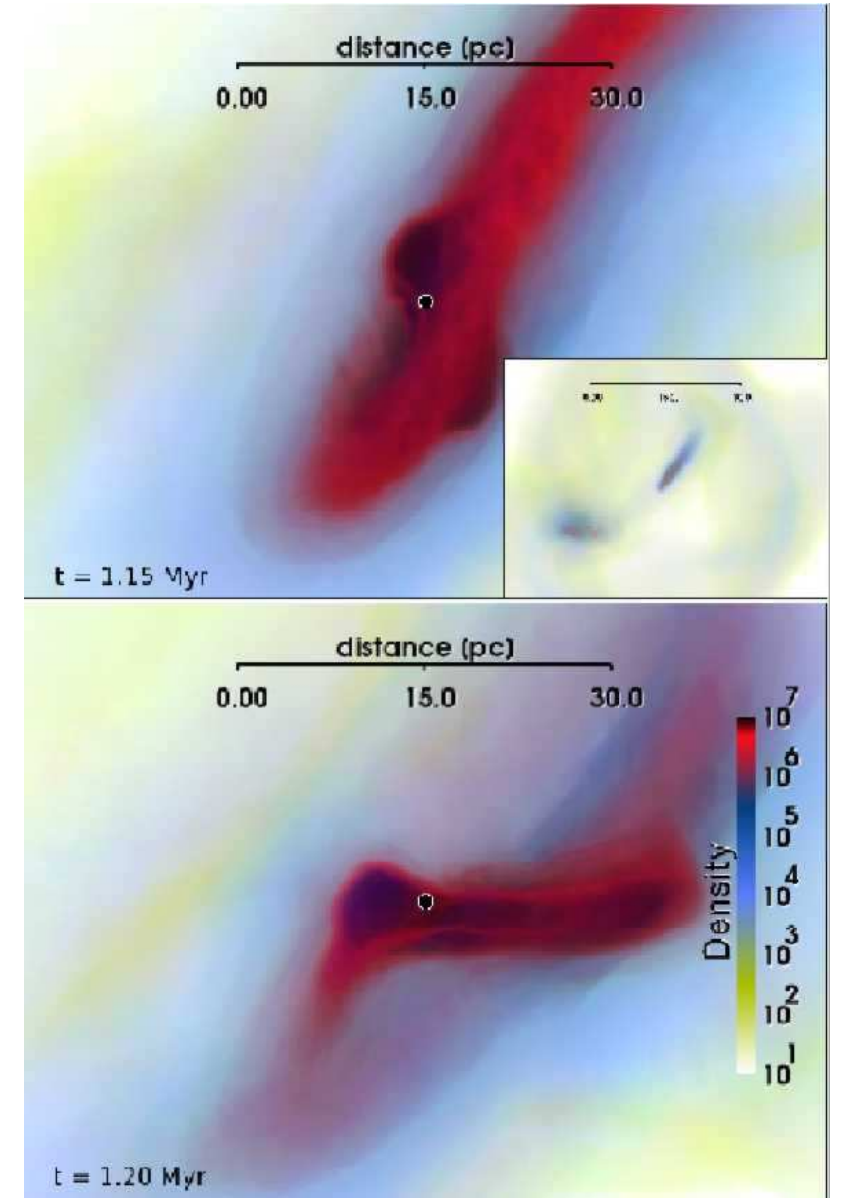

FIG. 10.- 3D volume-rendering of the gas density before (top) and after (bottom) the second angular momentum shift in the fiducial run referred to in the text (and in Figures 8 and 9). The inset in the top panel shows the large clump developing at a few hundred parsecs that is influencing the dynamics of the inner circumnuclear disk. The circle in the center of each panel corresponds to the position of the black hole particle. This figure is best viewed in color.

as it reaches the center of the circumnuclear region, a corresponding temporary displacement of the black hole particle is expected, similar to the earlier displacement. The black hole particle is likely to be displaced when the in-falling gas mass is comparable to or greater than the black hole mass and the mass of the gas disk interior to the in-falling clump. However, a displacement has not yet occurred by the end of the simulation. Similar angular momentum flips occur over the course of runs Z4L20.BH3, Z4L20.BH10, and Z4L20.OT (not shown), often corresponding to coincident displacements of the black hole particle.

The $z=3$ simulation shows more scatter at $100 \mathrm{pc}$ than the $z=4$ simulation. There is a rapid shift in the direction of the angular momentum vector of $\sim 30-45^{\circ}$ beginning at $\sim 0.35 \mathrm{Myr}$ in the $z=3$ simulation. The shift is less dramatic than both of those observed in the $z=4$ simulation. There is no corresponding black hole particle displacement, likely because the mass of in-falling clump is small compared to the mass of the disk (and the black hole). The orientation of the disk appears to be more coherent between the different scales in $z=3$ simulation than in the $z=4$ simulation. This may be a result of the circumnuclear disk being more massive at lower redshift. In broad terms however, the behavior of the angular momentum vector in each of the simulations consistently shows that the gas delivered to the SMBH may have varying angular momentum, which can shift suddenly as massive clumps of gas develop and move through the disk. The properties of the gas that is ultimately accreted may then determine the spin and subsequently the mode of feedback produced by the SMBH.

\section{DISCUSSION AND CONCLUSIONS}

We have studied the transport of gas in the circumnuclear region of a disk galaxy within a cosmological simulation at different redshifts, for different SMBH masses, and for a run including an approximation of optically thick cooling. The mass accretion rate is not steady, but fluctuates randomly and substantially, the accretion rate through the circumnuclear disk being almost as often negative as positive. This result is consistent with that of Hopkins \& Quataert (2009), who find a large amount of variation in the instantaneous accretion rates in their galaxy simulations on scales $<1 \mathrm{kpc}$. We find that there is no preferred timescale for accretion over the course of the $\sim$ million years spanned by each of the simulations. The flat slope of the Fourier transform of the accretion rate characterizes the fluctuations as a function of timescale, revealing the stochastic nature of accretion in the simulations. This complex and chaotic behavior is apparent at each of the three redshifts explored in detail here: $z=3,4$, and 6 , as well as in $z=4$ simulations containing black holes with larger masses, and the $z=4$ run including optically thick cooling.

The dynamic and turbulent nature of the simulated circumnuclear disk is not well modeled by Bondi accretion, even when using a modified prescription that takes into account the vorticity and turbulent properties of the gas. Bondi prescriptions do not take into account the effect of the disk's self-gravity. Additionally, the modified Bondi accretion rates, estimated from the properties of the disk in the highest-resolution part of the simulation (where the density rises steeply with decreasing radius), are highly super-Eddington on the smallest scales (assuming a radiative efficiency of 0.1 ). As the Eddington rate provides an upper limit for the accretion rate in the presence of radiative feedback from the SMBH, highly super-Eddington rates are not likely to endure in nature.

The Eddington rate (or a few times the Eddington rate) is set as an upper limit to the accretion rate in many of the simulations that employ Bondi type prescriptions to model accretion (e.g., Springel et al. 2005a; Di Matteo et al. 2005; Li et al. 2007; Di Matteo et al. 2008). On scales corresponding to the spatial resolution of those simulations $(\gtrsim 100 \mathrm{pc}$ ), the accretion rates predicted by the modified Bondi prescription in the present simulations are closer to the Eddington limit than they are at smaller scales. However, black holes in other simulations spend the majority of time accreting well below this limit, at substantially sub-Eddington rates. The difference between the rates determined here and those of other simulations is even larger when comparing with prescriptions that multiply the Bondi accretion rate by a large factor to approximate the effects of the small scale physics, since no such factor has been included in our calculations (see discussion of the parameter $\alpha$ 
Booth \& Schave 2009; Johansson et al. 2009a, b). However, it is not straightforward to make a direct comparison between accretion rates in the present simulations and the rates in other simulations that contain prescriptions for AGN feedback. As shown in galaxy merger simulations (e.g., Springel et al. 2005a; DeBuhr et al. 2009), the presence of AGN feedback regulates black hole growth by forcing gas out of the circumnuclear region. Future work incorporating AGN feedback into the present simulations will address this issue.

The absence of AGN feedback in our simulations may result in an excessively dense circumnuclear gas disk, which corresponds to large, super-Eddington accretion rates within the Bondi prescription. Allowing the black hole particle to grow in situ in our simulations, or using a more physically motivated recipe for star formation in the zoom-in simulation would further change the distribution of mass in the circumnuclear gas disk. The circumnuclear disk is self-gravitating, almost certainly behaving outside the Bondi regime, even with the modifications of Krumholz et al. (2006). This result reinforces the need for high-resolution simulations incorporating detailed gas dynamics and radiative processes, in order to truly model the complicated dynamics on small scales, which ultimately govern accretion onto SMBHs.

The chaotic behavior of the disk may be consistent with models suggesting that stochastic accretion of molecular clouds from the circumnuclear region of the galaxy can power low-luminosity AGNs (Hopkins \& Hernquist 2006). This manner of accretion may determine the spin of the black hole. We find that the angular momentum vector on scales $\lesssim 100$ pc can vary substantially from the direction of angular momen- tum on kiloparsec scales. In each simulation, the axis of the disk is closely aligned with the axis on kiloparsec scales at the beginning of the zoom-in simulation, but slowly over time shifts direction as the circumnuclear disk dynamically evolves.

The angular momentum can be misaligned with the rest of the circumnuclear disk, resembling the stochastic fueling scenarios mentioned in Section 5. As is observed in several of the simulations presented here, clumps of gas develop in the circumnuclear disk, which change the angular momentum of the gas that ultimately feeds the accretion disk. The change can occur as clumps fall into the center or as larger clumps develop within the disk and change the potential. However, as cautioned above, the simulation does not yet include feedback from an accreting black hole, which will heat the gas and drive out some of the material in the circumnuclear disk. Future work including more realistic physics in the simulations will allow us to constrain better the effects of accreting gas on the properties of the SMBH and the feedback it produces.

The authors thank the anonymous referee for constructive comments and suggestions. This work was supported in part by the DOE and the NASA grant NAG 510842 at Fermilab and by the NSF grants AST-0134373, AST-0507596, and AST-0708607. Supercomputer simulations were run on the IBM P690 array at the National Center for Supercomputing Applications and San Diego Supercomputing Center (under grant AST-020018N), as well as on the Joint Fermilab-KICP Supercomputing Cluster (supported by grants from Fermilab, the Kavli Institute for Cosmological Physics, and the University of Chicago).

\section{REFERENCES}

Abramowicz, M. A., \& Zurek, W. H. 1981, ApJ, 246, 314

Barnes, J. E., \& Hernquist, L. 1992, ARA\&A, 30, 705

Begelman, M. C., \& Shlosman, I. 2009, ApJ, 702, L5

Berti, E., \& Volonteri, M. 2008, ApJ, 684, 822

Bondi, H. 1952, MNRAS, 112, 195

Bondi, H., \& Hoyle, F. 1944, MNRAS, 104, 273

Boone, F., et al. 2007, A\&A, 471, 113

Booth, C. M., \& Schaye, J. 2009, MNRAS, 398, 53

Croft, R. A. C., Di Matteo, T., Springel, V., \& Hernquist, L. 2009, MNRAS, 400, 43

Croton, D. J., et al. 2006, MNRAS, 365, 11

DeBuhr, J., Quataert, E., Ma, C., \& Hopkins, P. 2009, arXiv:astro-ph/0909.2872

Di Matteo, T., Colberg, J., Springel, V., Hernquist, L., \& Sijacki, D. 2008, ApJ, 676, 33

Di Matteo, T., Springel, V., \& Hernquist, L. 2005, Nature, 433, 604

Englmaier, P., \& Shlosman, I. 2004, ApJ, 617, L115

Escala, A. 2007, ApJ, 671, 1264

Ferland, G. J., Korista, K. T., Verner, D. A., Ferguson, J. W., Kingdon, J. B., \& Verner, E. M. 1998, PASP, 110, 761

Ferrarese, L., \& Merritt, D. 2000, ApJ, 539, L9

Fukuda, H., Habe, A., \& Wada, K. 2000, ApJ, 529, 109

García-Burillo, S., Combes, F., Schinnerer, E. a nd Boone, F., \& Hunt, L. K. 2005, A\&A, 441, 1011

Gebhardt, K., et al. 2000, ApJ, 539, L13

Gnedin, N. Y., Tassis, K., \& Kravtsov, A. V. 2009, ApJ, 697, 55

Hernquist, L. 1989, Nature, 340, 687

Hopkins, P. F., Cox, T. J., Kereš, D., \& Hernquist, L. 2008a, ApJS, 175, 390

Hopkins, P. F., \& Hernquist, L. 2006, Astrophys. J. Suppl., 166, 1

Hopkins, P. F., Hernquist, L., Cox, T. J., Di Matteo, T., Martini, P., Robertson, B., \& Springel, V. 2005, ApJ, 630, 705

Hopkins, P. F., Hernquist, L., Cox, T. J., \& Kereš, D. 2008b, ApJS, 175,356

Hopkins, P. F., Hernquist, L., Cox, T. J., Robertson, B., \& Krause, E. 2007, ApJ, 669, 45
Hopkins, P. F., \& Quataert, E. 2009, arXiv:astro-ph/0912.3257

Hoyle, F., \& Lyttleton, R. A. 1939, in Proceedings of the Cambridge Philisophical Society, Vol. 35, Proceedings of the Cambridge Philisophical Society, 405-+

Jiang, L., Fan, X., Ivezić, Ž., Richards, G. T., Schneider, D. P., Strauss, M. A., \& Kelly, B. C. 2007, ApJ, 656, 680

Johansson, P. H., Burkert, A., \& Naab, T. 2009a, ApJ, 707, L184

Johansson, P. H., Naab, T., \& Burkert, A. 2009b, ApJ, 690, 802

Kauffmann, G., \& Haehnelt, M. 2000, MNRAS, 311, 576

Kawakatu, N., \& Wada, K. 2008, ApJ, 681, 73

Kennicutt, Jr., R. C. 1998, ApJ, 498, 541

King, A. R., \& Pringle, J. E. 2006, MNRAS, 373, L90

-. 2007, MNRAS, 377, L25

King, A. R., Pringle, J. E., \& Hofmann, J. A. 2008, MNRAS, 385 1621

Kormendy, J., \& Kennicutt, Jr., R. C. 2004, ARA\&A, 42, 603

Kormendy, J., \& Richstone, D. 1995, ARA\&A, 33, 581

Kravtsov, A. V. 1999, PhD thesis, AA(NEW MEXICO STATE UNIVERSITY)

Kravtsov, A. V., Klypin, A., \& Hoffman, Y. 2002, ApJ, 571, 563

Kravtsov, A. V., Klypin, A. A., \& Khokhlov, A. M. 1997, ApJS, 111,73

Krumholz, M. R., McKee, C. F., \& Klein, R. I. 2005, ApJ, 618, 757

-. 2006, ApJ, 638, 369

Levine, R. 2008, PhD thesis, University of Colorado at Boulder

Levine, R., Gnedin, N. Y., Hamilton, A. J. S., \& Kravtsov, A. V. 2008, ApJ, 678, 154

Li, Y., et al. 2007, ApJ, 665, 187

Lindt-Krieg, E., Eckart, A., Neri, R., Krips, M. a nd Pott, J.-U.,

García-Burillo, S., \& Combes, F. 2008, A\&A, 479, 377

Lomb, N. R. 1976, Ap\&SS, 39, 447

Lynden-Bell, D. 1969, Nature, 223, 690

Magorrian, J., et al. 1998, AJ, 115, 2285

McCarthy, I. G., et al. 2009, arXiv:astro-ph/0911.2641

Nayakshin, S., \& King, A. 2007, arXiv:astro-ph/0705.1686 
Press, W. H., Teukolsky, S. A., Vetterling, W. T., \& Flannery, B. P. 1992, Numerical recipes in C. The art of scientific computing (Cambridge: University Press, -c1992, 2nd ed.)

Proga, D., \& Begelman, M. C. 2003, ApJ, 582, 69

Regan, J. A., \& Haehnelt, M. G. 2009, MNRAS, 396, 343

Regan, M. W.. \& Teuben, P. J. 2004, ApJ, 600, 595

Riffel, R. A., Storchi-Bergmann, T., Winge, C., McGregor, P. J., Beck, T., \& Schmitt, H. 2008, MNRAS, 385, 1129

Roberts, Jr., W. W., Huntley, J. M., \& van Albada, G. D. 1979, ApJ, 233, 67

Saitoh, T. R., \& Wada, K. 2004, ApJ, 615, L93

Salpeter, E. E. 1964, ApJ, 140, 796

Shlosman, I., Frank, J., \& Begelman, M. C. 1989, Nature, 338, 45

Sijacki, D., Springel, V., Di Matteo, T., \& Hernquist, L. 2007, MNRAS, 380, 877

Sijacki, D., Springel, V., \& Haehnelt, M. G. 2009, MNRAS, 400, 100

Silk, J., \& Rees, M. J. 1998, A\&A, 331, L1

Soltan, A. 1982, MNRAS, 200, 115

Springel, V., Di Matteo, T., \& Hernquist, L. 2005a, ApJ, 620, L79
-. 2005b, MNRAS, 361, 776

Storchi-Bergmann, T., Dors, Jr., O. L., Riffel, R. A. a nd Fathi, K., Axon, D. J., Robinson, A., Marconi, A., \& Östlin, G. 2007, ApJ, 670, 959

Tremaine, S., et al. 2002, Ap.J, 574, 740

Volonteri, M., Sikora, M., \& Lasota, J.-P. 2007, ApJ, 667, 704

Wada, K. 2001, ApJ, 559, L41

Wada, K. 2004, in Coevolution of Black Holes and Galaxies, ed. L. C. Ho, $186-+$

Wada, K., Meurer, G., \& Norman, C. A. 2002, ApJ, 577, 197

Wada, K., \& Norman, C. A. 1999, ApJ, 516, L13

-. 2001, ApJ, 547, 172

‥ 2007, ApJ, 660, 276

Wang, J., et al. 2009, ApJ, 697, L141

Wise, J. H., Turk, M. J., \& Abel, T. 2008, ApJ, 682, 745

Wong, T., \& Blitz, L. 2002, ApJ, 569, 157

Wyithe, J. S. B., \& Loeb, A. 2003, ApJ, 595, 614

Young, J. S., Allen, L., Kenney, J. D. P., Lesser, A., \& Rownd, B. 1996, AJ, 112, 1903 